Available online at_www.iponlinejournal.com

\title{
Behavioural medicine for oral psychosomatic disorders
}

\section{Sumit Bhateja $^{1^{*}}$, Harshita Gupta ${ }^{2}$, Shaifaly Chouhan ${ }^{3}$, Medha Sansanwal ${ }^{4}$, Geetika Arora ${ }^{5}$}

${ }^{\mathbf{1}} \mathrm{HOD},{ }^{\mathbf{2}-\mathbf{4}}$ Dental surgeon, ${ }^{\mathbf{5}}$ Reader, ${ }^{\mathbf{1}}$ Dept. of Oral Medicine \& Radiology, ${ }^{\mathbf{5}}$ Dept. of Public Health Dentistry, ${ }^{\mathbf{1 - 4}}$ Manav Rachna Dental College, Faridabad, Haryana, Inderprastha Dental College, Sahibabad, Uttar Pradesh, India

\begin{abstract}
There are many patients who have 'medically and psychiatrically unexplained symptoms' in clinical dentistry. These symptoms are called "Oral Psychosomatic Disorders". It is not uncommon to see the patients with these conditions, so there is a growing need for proper management of these disorders from both sides of doctors and patients. So it becomes important to have identity as a dentist on practice of psychosomatic dentistry. This article reviews various psycho therapies for stress management towards establishment of 'Psychosomatic Dentistry'.
\end{abstract}

Keywords: Psychosomatic disorders, Emotional stress, Behavioral medicine.

\section{Introduction ${ }^{1,2}$}

Psychosomatics or the psycho physiological problem (mind - body relationship) is a question that has been asked for thousands of years. The term "psychosomatic" was first used by Heinroth (1818) when he discussed psychosomatic aspects of insomnia and was later popularized by German psychiatrists Jacobi \& Nasse. The psychoanalyst Franz Alexander (1935), considered to be the founder of psychosomatic medicine, proposed a theoretical model explaining the mechanism of psychosomatic disorders, which underlies much of clinical work \&research till date.

The American Psychiatric Association (1952) in its standard nomenclature recognized the ambiguity in the use of word "psychosomatic" \& coined the designation "psycho physiologic autonomic \& visceral disorder" for the symptoms that are seen due to chronic exaggerated state of normal physiological expression of emotions; such long term physiological $\&$ visceral states may eventually lead to structural changes in various organs.

"Psychosomatic medicine" is an area of scientific investigation concerned with the relation between psychological factors and physiological phenomena in general and disease pathogenesis in particular \&oral psychosomatic disorders which constitute an integral part.

We might also mention a different view of psychosomatics: understanding psychosomatics as $a$ psychosomatic problem i.e. astate of mind, mood, situation or long-term psychological trouble, situations of conflict are reflected in physical health. This involves psychological situations that are difficult to cope with. Or we can talk about a somatopsychic problem i.e. a sudden change in the physical condition, for example, toothache; physical attack causes secondary psychological problems. "Behavioral medicine" is the field concerned with the development of behavioral-science knowledge and techniques relevant to the understanding of physical health and illness and the application of this knowledge and these techniques to diagnosis, prevention, treatment and rehabilitation. Psychosomatic medicine has tended to emphasize etiology and pathogenesis, and behavioral medicine treatment and prevention, then each has contributed to a comprehensive view of disease, and ideally there should be no conflict between them (e.g., understanding etiology and pathogenesis is essential for rational treatment and prevention).

The best psychiatric approach is to construct a life chart for an individual. The determinants of stress as a

*Corresponding Author: Sumit Bhateja, Dept. of Oral Medicine \& Radiology, Manav Rachna Dental College, Faridabad, Haryana, India

Email: bhateja.sumit@gmail.com 
breaking point on body is patients own physiological vulnerability, constitutional make up \& genetic predisposition. There are many theories to explain the basis of psychosomatic disorders. They are based on concept of emotional stress on body system which is defined as physical, mental or emotional response to events that causes bodily or mention. So this article reviews different approaches to manage psychological stress.

\section{Illness as a human story3}

Each illness, each serious life-changing situation becomes a part of the human story; the illness is Us, it is not we that 'have an illness'. Everything we experience remains in us as annual rings on a tree. Paradoxically, many times an illness is also able to cure a person. Each emotional reaction is also at the same time a physical reaction; each physical reaction is also at the same time a psychological reaction. The body and its expression is a structured history of an individual, the body is an expression of the psychological reaction (W. Reich).

We are always in the place we are just thinking of (even if we are some where else). The question is whether we want to be there. We can talk about the emotional response of the body. By the way, the recent findings on so called mirror neurons in the brain tells us that one needs only look at somebody who is doing some thing, and simultaneously, mirror neurons activate our brains, even before we are able to realize it, in such a way that we ourselves are performing this activity even if we are not doing anything. Therefore, it is more appropriate, and more accurate, to always talk about interaction and not a subsequent reaction.

Vague statements about biopsychosocial causes are still shackled to a linear (hierarchical) approach, which instead of a systematic approach, involves an effort to ascribe traditional terminology to the chain of links that make up the whole. In practice, interest is divided as follows: bio (medical domain), psycho (psychological domain), and social (sociological domain, domain of social sciences). However, psychosomatics is valid in practice, as the title of this text also suggests. People do not talk much about the social anchoring of a human being, even if they regularly talk about our being biopsychosocially anchored. Let us add that psychosomatics, in the form of Cartesian dualism, is gradually being abandoned. Therefore, modern psychology is directed at a holistic approach to human beings in both health and illness. Every one of us always exists on a continuum between these two poles.

\section{Theoritical models- etiopathogenesis}

There exists a considerable disagreement about the nature of the predisposition for psychosomatic disorders \& the specific vulnerability that determines the choice of organ. The models fall into the following three categories:

\section{The emotional theory}

Postulates that emotional stress by accumulation leads - oral aggressive responses and guilt - anxiety overcompensation for oral aggression by the desire to give and accomplish- inhibition and failure of effort to give and accomplish - Psychosomatic reactions.

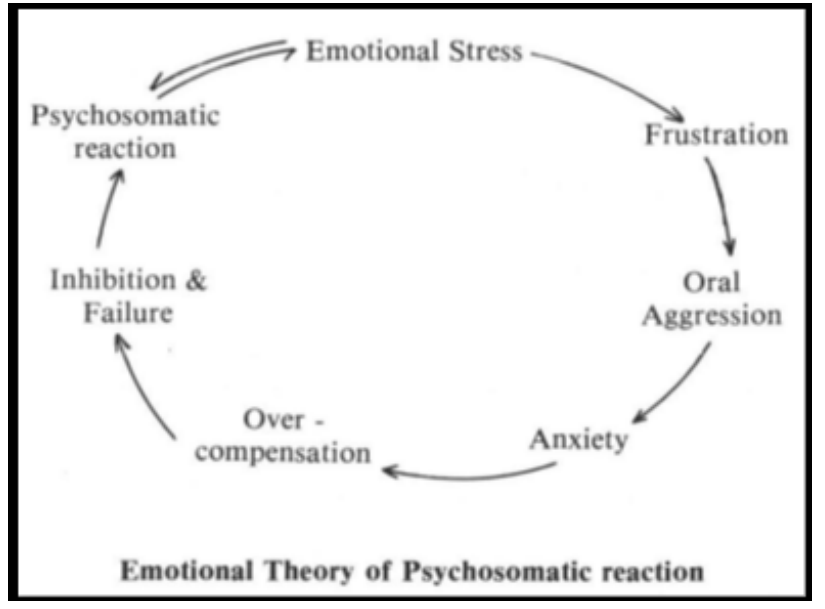

Fig. 1

Constituting a personality profile in patients with psychosomatic disorder, would include:

1. General adjustment in life i.e. education, work, record, income, vocational level, social relationships, sexual adjustments and attitude towards family.

2. Characteristic behavior pattern

3. Neurotic traits

4. Hobbies and interests

5. Life style immediately prior to onset of illness

6. Specific individual reaction to illness 
7. Focal conflict area and characteristic reaction.

The psychosomatic formula is based on certain criteria:

a. Emotion as a precipitating factor causing remission and relapse.

b. Personality type: There was a high association of certain personality type with specific illness type.

c. Time specificity or relationship with other psychosomatic reaction in the same individual at same time of illness or some other specific time during life history.

d. Family history: There is a high percentage of the same disorder occurring in the individual in the parents or the relatives and siblings.

e. Sex ratio: There is a disproportion in sex ratio, distribution, thus ulcerative colitis is higher in females 3:1 respectively.

f. Periodicity: These illnesses occur in phases characterized by certain time charts pattern.

The effects of emotion on bodily functions are familiar to everybody and people express themselves in psychosomatic language describing the emotional components in their subjective experience symbolizing the relationship between emotions and bodily changes, i.e. it gives me a headache or it make be sick etc.

Canon (1939) studied the effect on autonomic system and bodily changes in pain, fear and rage and suggested that the sympathetic stimulation prepares the body for fight or flight indicating the importance of autonomic nervous system and the medulla of supra renal glands in these reactions. Later Wolff \& Wolff confirmed his findings through laboratory confirmations illustrating effect of emotion on body organ.

\section{Selye's theory of stress adaptation Syndrome: ${ }^{4}$}

This is based on the adrenocortical response to physical and emotional stimuli as producing stress. The concept of stress in the psychosomatic sense is regarded as the result of a frustrating experience which the individual is unable to influence or where there is a mental conflict expressed in somatic form.

\section{Adler theory of "organ inferiorty"(1924):}

Postulates an underlying weakness of the organ concerned with the continuous stress in relation to it, i.e. a relationship between stressful life situation and exacerbation of organ reaction to it.

Thus there are several stages in response to stress:

1. The organism can meet the need of the stress

2. The organism can meet the need but only for a period which may not be sufficient

3. The organ can meet the need without abandoning at least for the time being, the effort to pursue or evade some other relationship.

4. The organism cannot meet the need at all.

5 .

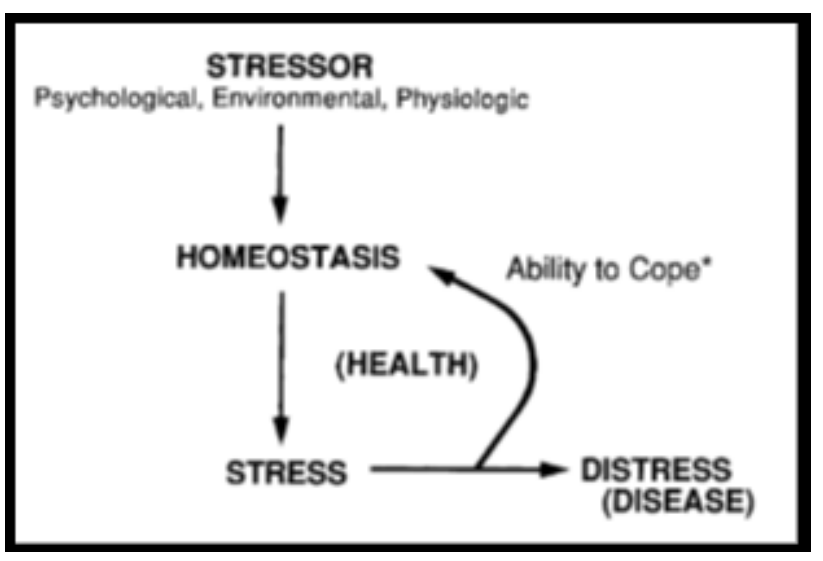

Fig. 2

Classification of oral psychosomatic disorders: ${ }^{7,8}$ McCarthy PL and Shklar G (1980)

1. Oral Psychosomatic Disease

a. Lichen Planus

b. Aphthous Stomatitis

c. Glossitis \& Stomatitis areatamigrans

2. Oral Diseases in which Psychological factors may play some etiologic role

a. Erythema Multiforme

b. Mucous Membrane Pemphigoid

c. Chronic Periodontal Disease

3. Oral Infections in which emotional stress serves as a predisposing factor

a. Recurrent Herpes Labialis

b. Necrotizing Gingivitis

4. Oral Disease induced by Neurotic Habits

a. Leukoplakia

b. Biting Of Oral Mucosa (Self-Mutilation)

c. Physical / Mechanical Irritation 
d. Dental / Periodontal Disease produced by bruxism

\section{Neurotic Oral Symptoms}

a. Glossodynia (Glossopyrosis)

b. Dysgeusia

c. Mucosal Pain

\section{Bailoor \& Nagesh (2001)}

\section{Pain Related Disorders}

a. Myofacial Pain Dysfunction Syndrome

b. Atypical Facial Pain

\section{Disorders Related To Altered Oral Sensation}
a. Burning Mouth Syndrome
b. Idiopathic Xerostomia
c. Idiopathic Dysguesia

\section{Miscellaneous}
a. Oral Lichen Planus
b. RecurrentApthous Ulcers
c. Psoriasis
d. Erythema Multiforme
e. Cancerophobia
f. ANUG
g. Anorexia Nervosa
h. Bruxism

\section{Psychosomatics in oral medicine: ${ }^{9}$}

Oral changes with psychosomatic etiology are still an insufficiently confirmed and investigated subgroup of psychosomatic diseases which have long been known in medicine. Many of them, which are believed to be of psychosomatic character, still do not have sufficient explanation of their etiology, or it is considered to be multicausal. In daily practice dentists frequently come across patients showing signs of different mental disorders, such as anxiety, fear and various forms of neuroticism. Thus, the above facts implicate the need to examine the possibility of a relationship between oral and mental health, and particularly whether there are long term negative mental changes and/or certain personality traits which may have an effect on the oral mucous membrane via psychosomatic mechanism.

It is beyond doubt that stress plays a role in etiology of oral psychosomatic disorders, subsequent section deals with various therapies towards management of psychological stress.

\section{Therapies for management psychological stress: ${ }^{10}$}

The approaches we discuss here can be conducted or supervised by oral physicians in their day to day practice; psychologists and other mental health professionals may help patients prepare for treatment or cope with its after effects.

Approaches / Therapies can be classified as:

\section{Biomedical therapies}

a. Electroconvulsive/Electroshock therapy

b. Psychosurgery

c. Chemotherapy

\section{Psychodynamic therapies}

a. Freud's traditional psychoanalysis

b. Traditional vs contemporary psychoanalysis

c. Dissident Theorists \& their techniques: JUNG, ADLER \& HORNEY

\section{Humanistic \& existential therapies}

a. Client centered therapy

b. Gesalt therapy

c. Existential therapy

4. Behavioural therapies
a. Operant conditioning technique
b. Classical conditioning technique
c. Modeling technique

5. Coagnitive therapies

a. Ellis rational emotional therapy

b. Beck's cognitive therapy

c. Meichenbaum'sself instructional training

\section{Group therapies}
a. Family therapy
b. Group therapy

\section{Behavioural medicine}
a. Relaxation training
b. Hypnosis
c. Biofeedback

We will begin our discussion of therapy by focusing on each of therapies:

\section{Biomedical therapies: ${ }^{11}$}

Electroshock therapy(EST) is reserved for severely depressed patients. Electrodes are attached to one or both temples \& electricity (70-160 volts for 0.1-0.5 seconds) is passed directly through the brain. Jolts of electricity enhance feelings of well being by producing a cortical orgasm or merely a placebo effect. 
Psychosurgery, like prefrontal lobectomy, is the removal of brain tissue from the prefrontal cortex and prefrontal lobotomy, the severing of connections between the prefrontal cortex and the rest of brain.

Chemo therapyis treatment with chemical substances or drugs. The class of drugs, known as antipsychotics, reduces agitation, tension and combativeness; decrease or eliminate delusions and hallucinations; and improve social behavior and sleep patterns. Common examples are phenothiazenes, tricyclics \& monoamino oxidase (MAO) inhibitors.

Bright side of chemotherapy is that it does relieve symptoms but on darker side these medications are habit forming \& carry risk of some serious side effects like tardive dyskinesia with phenothiazenes. Therapies especially EST \& surgery should be considered when all other therapies fail to respond because they are considered controversial \& their use has declined in recent times.

\section{Psychodynamic therapies ${ }^{12-14}$}

1. Freud's traditional psycho analysis states that we all experience certain sexual and aggressive urges springing from our is that clash with barriers imposed by our egos and thus are incorporated into our superegos. This creates a vague fear in us called anxiety. To counter anxiety, our body triggers abnormal neurotic behaviors that distort our sense of reality and impair our ability to function. To counter this, Freud started analysis by developing insight, a deep understanding of repressed feelings and conflicts. The analyst traces the connection between the neurotic behavior and psychological process and gradually leads the patient to understand and accept their neurotic behavior to liberate them from neurosis. This can be done via:
a. Coping with Resistance, Transference, and Countertransference
b. Insight, "Working Through", and Termination
c. Probing the Unconscious: Free Association and Dream Analysis

2. Traditionalversus Contemporary Psychoanalysis states the difference between the thinking of traditional therapists and contemporary therapists. Traditional therapy involves five 50 minutes sessions per week for several years but contemporary therapists work out time limits with their patients and treat them as partners to stop them from getting dependent on therapy.

3. Dissident Theorists and their Techniques:

a. Jung's approach known as analytical therapy placed less emphasis on sexual impulses and instead focused on each person's need for individuation i.e. "becoming a single, homogenous being coming to selfhood or self realization. He used word association and dream analysis.

b. Alfred Adler's analysis known as individual therapy placed special emphasis on social and interpersonal factors like patient's need for meaning, personal freedom, and a fulfilling ' style of life''.

c. Karen Horney's analysis emphasized on social factors. She helped patients identify their maladaptive interpersonal strategies and their reasons for using them; then tried to lead them towards more constructive interpersonal styles and greater self-reliance.

\section{Humanistic and existential therapies}

1. Client-centered Therapy was therapy Carl Rogers. He believed mal adjustment occurs when; patients have discrepancies between their real and ideal selves; these discrepancies become sizable and painful. Patient is called client to make him comfortable as if paying for his service. The therapist supports client's effort at self-discovery and growth without judgmental or pushy. For this type of therapy therapist must be empathic, sensitive, positive, never criticizing, always accepting and never disapproving. The client centered therapist uses nondirective techniques like reflection of feeling.

2. Gestalt Therapy is designed to make people "whole" by encouraging them to relieve their pentup feelings, accept responsibility for themselves, and focus on the present. Therapist encourages client to use I statements like "I feel...", "I am..." etc. These statements help patient to confront their present feelings instead of ruminating in the past. 
3. Existential Therapy is developed by a group of therapists like Viktor Frankl and Rollo May that all focused on existential themes. They tried to make the client aware that they always have choices and thus control over their fate. They prod people into ceasing control of their lives by making them see that their problems are a result of their own choices and can be resolved by making healthier choices.

\section{Behavioral Therapies}

1. Instrumentalor Operant Conditioning techniques essentially focus in the concept of "payoffs". Client works with the therapist to develop a reward system wherein he is rewarded for desired behavior to stimulate and maintain such behavior while extinguishing undesired behavior. This is done in several steps: functional analysis of behavior; identifying positive and negative reinforcers; extinction; differential reinforcement; shaping and token economies; punishment and covert sensitization.

2. Classical conditioning techniques includes therapy when two stimuli are paired. A conditioned stimulus produces no response before conditioning; but after it hasn't paired with unconditioned stimulus a number of times, it begins to produce a response often similar to response previously only given to unconditioned stimulus. These include systematic desensitization; flooding and aversion therapy.

3. Modeling techniques are based on cognitive learning known as observational learning. Often used to treat phobias, it helps the client acquire new behavior by either providing information on how to perform the new behavior or by giving evidence of the "do-ableness" of the behavior. A person's sense of self efficacy can be increased by this.

\section{Cognitive approaches}

1. Ellis's rational-emotive therapy (RET) is designed to reveal and breakdown irrational beliefs that lead to distress. The therapist probes into client's behavior and belief system for irrational beliefs that may have been unthinkingly accepted for years and may be causing suffering. By uncovering such damaging cognitions, stating them clearly, and exposing their irrationality, the RET therapist tries to help clients structure a more realistic, less punishing belief system.

2. Beck's Cognitive Therapy is designed primarily for the treatment of depression using "pointed, but friendly questions" to root out depressed people's faulty "depressogenic cognitions". They also initiate behavior change and insure that the depressed people will engage in rewarding activities. Thus, it helps in altering depression generating cognitions.

3. Meichenbaum's self-intructional training uses self instructional training to help clients replace their maladaptive cognitions with rational, positive thoughts, particularly in stressful situations.

\section{The emerging field of behavioral medicine}

1. Relaxationtraining is used for systematic desensitization of fears; chronic pain etc. It can be supplemented by guided imagery. One of use of relaxation technique is in treatment of chronic pain. Many kinds of pain involve muscle tension which is incompatible with relaxation. It is based on principle of reciprocal inhibition which says that two incompatible responses cannot occur at the same time and that the stronger of two will replace the weaker, thus fear \& anxiety weaken if patient remains relaxed. This principle can be put to use by training people to relax those muscles contributing to pain. The therapist uses progressive muscle relaxation, with training gradually zooming in on the shoulders, neck and face.

2. Hypnosis is a deep trance like state in which the clients become extremely suggestible. It can be used to get clients to comply with direct suggestions for behavior change, and to alleviate nausea and vomiting, especially in cancer patients. Heightened suggestibility is used to get patients to comply with direct suggestions for behavior change.

3. Biofeedback is a procedure in which people learn to modify their responses such as heart rate and body temperature through instrumental 
conditioning. It is effective in treatment of cardiac arrhythmia, Raynaud's syndrome etc. By providing feedback information to reward learners for specific physiological responses, such as speeding up their heart rate or expanding certain blood vessels.

However some explanations propose that biofeedback is effective because it teaches people skills in generalized relaxation that lowers overall sympathetic nervous system activity and in turn moves many physiological processes away from over activity; or that biofeedback teaches people to control internal processes by using self statements or specific mental images to trigger specific physiological reactions.

\section{Therapy for groups}

1. Family therapy involves interpretation and treatment of psychological problems in systemic fashion by involving entire family operating as a system. Disturbed behavior by one family member may be stimulated, or worsened, by disturbed relationship in the family as a whole. Therapists arrange family sessions to discuss problems. They emphasize psychodynamics; that is they try to uncover underlying motives and conflicts within the family and stimulate insight to teach how to solve the problems. They may also use humanistic-existential approaches or behavioral frameworks.

2. Group Therapy is intended to capture the essence of "real life" in social context. It is attractive to people who are not severely distressed but want to gain insight of increased sensitivity through structured interaction with others. The group consists of 5-15 people, have 1 or 2 leaders or trainers who tried to create an atmosphere in which emotions and feeling are stressed.

\section{Community Psychology: Outreach and prevention in real life settings}

1. Solving problems were they happen involves getting into settings where problems have already begun so that psychologist can see problems as they unfold naturally, design interventions to fit the settings, and assist the impact of those interventions.
2. Prevention seeks to reduce psychological distress in problems by finding and attacking their causes, this is primary prevention. Secondary prevention identifies problems just as they begin to develop and stop them from becoming severe. Tertiary prevention is aimed in minimizing future difficulties in people already identified as having severe disorders or problems.

3. Social support: Person-to-Person Helping involves providing social support by known professionals to help people cope with stressful life events and can sometimes hold down the intensity of psychological disorders such as depression.

\section{Conclusion}

Psychosomatic disorders are diseases that involve both mind and body; or diseases that are physical but get worsened by psychological factors like stress and anxiety. Such disorders are not easily treated by just medicine and require a lot of time, patience and creativity by the doctor. Over the past century, a lot of research has been conducted to treat these disorders. This research comes under the umbrella of Behavior Medicine. Behavior medicine has evolved with each passing generation to create a more suitable, comfortable and better treatment for the patient. To decide the best treatment, the etiopathogenesis and diagnosis of the disease should be done correctly.

Oral psychosomatic disorders like lichen planus, leukoplakia, stomatitis etc are very common but their appropriate treatment often gets swept under the rug because of misinformation or ignorance of the doctor. Behavior therapies ranging from biomedical to psychodyanamic should be studied and applied correctly to give each patient appropriate care and to ensure good oral and over-all health.

\section{Source of Funding}

None.

\section{Conflict of Interest}

None. 


\section{References}

1. Grof S.Physical Manifestations of Emotional Disorders Observations from the Study of Non-Ordinary States of Consciousness. Inner Door 1997;9(2):7-9

2. Schwartz GE, Weiss SM. What is Behavioral Medicine? Psychosom Med 1977;39(6):377-81.

3. JiříKučírek. 2006. Psychosomatic disorders and Children. Psychosomatics and children.

4. Tan S.Y, Yip A. Hans Selye(1907-1982): Founder of Stress Thoery. Singapore Med J 2018;59(4):170-1.

5. Solomon, M. Review of Study of organ inferiority and its psychical compensation: A contribution to clinical medicine. [Review of the book Study of organ inferiority and its psychical compensation: A contribution to clinical medicine. A. Adler]. J Abnormal Psychology 1917;12(5):348-51.

6. Peterson PK, Chao CC, Molitor T, Murtaugh M, Strgar F, Sharp BM. Stress and Pathogenesis of Infectious Disease. Rev Inf Dis 1991;13(7):4-9.

7. Kumar NN, Panchaksharappa MG, Annigeri RG. Psychosomatic Disorders: An Overview for Oral Physician. J Indian Acad Oral Med Radiol 2016;28(2):4-9.

8. Maheshwari TNU, Gnanasundaram N. Stress Related Oral Diseases: A Research Study. Int J Pharma Bio Sci 2010;1(3)
9. Richter I, Vidas I, TurËinoviÊ P. Relationship of Psychological Characteristics and Oral Diseases with Possible Psychosomatic Aetiology. Acta Stomat Croat 2003;37(1):35-9.

10. Morgan CT, King RA, Weisz JR, Schopler J. Introduction to Psychology. Seventh Edition. India: McGraw Hill Education. Chapter 16. Ther Psychol Distress 666-724

11. Wittkower ED. Treatment of Psychosomatic Disorders. Canada Med Assoc J 1964;90(18):1055-60.

12. Brown MA, Munford AM, Munford PR. Behavior Therapy of Psychological Disressin Patients after Myocardial Infarction or Coronary Bypass. J Cardiopulm Rehabil 1993;13;201-10.

13. Fava GA, Cosci F, Sonino N. Current Psychosomatic Practice. Psychotherapy and Psychosomatics. 2017;86(1): 3-30

14. Decot E. Therapeutic methods for psychosomatic disorders in oto-rhino-laryngology. GMS current topics in otorhinolaryngology, head and neck surgery, 4, Doc21. 2005.

How to cite this article: Bhateja $\mathrm{S}$, Gupta $\mathrm{H}$, Chouhan S, Sansanwal M, Arora G. Behavioural medicine for oral psychosomatic disorders. J Nutr, Metab Health Sci, 2019;2(3):71-8. 